The Astrophysical Journal, 236:L121-L125, 1980 March 15

(c) 1980. The American Astronomical Society. All rights reserved. Printed in U.S.A.

\title{
THE ISOTOPIC COMPOSITION OF GALACTIC COSMIC-RAY IRON NUCLEI
}

\author{
R. A. Mewaldt, J. D. Spalding, E. C. Stone, and R. E. Vogt \\ California Institute of Technology \\ Received 1979 October 9; accepted 1979 November 28
}

\begin{abstract}
We report high-resolution observations made in interplanetary space of 83-284 MeV per nucleon galactic cosmic-ray iron isotopes and directly establish that ${ }^{56} \mathrm{Fe}$ is the dominant cosmic-ray $\mathrm{Fe}$ isotope. We find the following percentage abundances for $\mathrm{Fe}$ at the cosmic-ray source: ${ }^{54} \mathrm{Fe}=$ $9(+8,-5) \%,{ }^{55} \mathrm{Fe} \leq 7 \%,{ }^{56} \mathrm{Fe}=91(+5,-11) \%,{ }^{57} \mathrm{Fe} \leq 8 \%$, and ${ }^{58} \mathrm{Fe} \leq 6 \%$. When compared to calculated nucleosynthesis yields and other observations, these results place significant constraints on the neutron excess of the environment where cosmic-ray Fe originates.
\end{abstract}

Subject headings: cosmic rays: abundances-nucleosynthesis

\section{INTRODUCTION}

Iron has long been recognized as one of the key elements for understanding the origin of galactic cosmic rays. Because ${ }^{56} \mathrm{Fe}$ has the largest binding energy per nucleon of the stable nuclei, it represents the end of the chain of nucleosynthetic products that can be formed by fusion reactions in stars. Theoretical studies (see, e.g., Hainebach et al. 1974) show that the relative abundances of the iron isotopes are sensitive to the environment in which stellar nucleosynthesis occurs. Solar system $\mathrm{Fe}$ is more than $90 \%{ }^{56} \mathrm{Fe}$, but over a wide range of stellar environments ${ }^{54} \mathrm{Fe}$ is expected to be the dominant iron isotope, although compositions of intermediate character are also possible. Recent observations (see, e.g., Garcia-Munoz, Simpson, and Wefel 1979; Greiner et al. 1979; Mewaldt et al. 1979a, 1980) have established that the ${ }^{22} \mathrm{Ne} /{ }^{20} \mathrm{Ne}$ ratio at the cosmicray source is $\sim 3$ times that measured in high-energy particles accelerated in solar flare events (Dietrich and Simpson 1979; Mewaldt el al. 1979b), suggesting that cosmic rays and solar system material may have different origins. If so, isotopic differences in Fe might also be expected.

Observations of the iron isotopes in cosmic rays have been limited by the experimental difficulties inherent in resolving adjacent isotopes that differ by only $\sim 2 \%$ in mass. Although a number of earlier studies (see, e.g., the reviews by Meyer [1975] and Waddington [1977]) have suggested that the isotopic composition of cosmicray iron may be characterized by nonsolar abundances of ${ }^{54} \mathrm{Fe}$ and/or ${ }^{58} \mathrm{Fe}$, there are also significant discrepancies between the various measurements. Recently, studies using improved balloon-borne instrumentation (Tarlé, Ahlen, and Cartwright 1979; Webber, Kish, and Simpson 1979; Young 1979) concluded that cosmicray iron is composed mainly of a single dominant isotope. Although the mass scale for these instruments was not calibrated in an absolute sense, supporting evidence was offered for a conclusion that ${ }^{56} \mathrm{Fe}$ is the dominant isotope, with only small amounts of ${ }^{54} \mathrm{Fe}$ and ${ }^{58} \mathrm{Fe}$, as in the solar system (see, e.g., Tarlé et al. 1979).
In this Letter, we report satellite observations of cosmic-ray iron isotopes that directly establish that ${ }^{56} \mathrm{Fe}$ is the dominant cosmic-ray $\mathrm{Fe}$ isotope. These observations cover an energy interval from 83 to $284 \mathrm{MeV}$ per nucleon, with a mass resolution of $\sigma=0.37 \mathrm{amu}$. We obtain finite source abundances for ${ }^{54} \mathrm{Fe}$ and ${ }^{56} \mathrm{Fe}$ and upper limits on the source abundances of ${ }^{55} \mathrm{Fe},{ }^{57} \mathrm{Fe}$, and ${ }^{58} \mathrm{Fe}$, which, when compared to calculated nucleosynthesis yields, place significant constraints on the environment where cosmic-ray $\mathrm{Fe}$ originates.

\section{OBSERVATIONS}

The observations reported in this study were made with the Caltech Heavy Isotope Spectrometer Telescope (HIST) on ISEE 3, during solar quiet-time periods between 1978 August 13 and December 1. The HIST telescope, described in detail by Althouse et al. (1978), consists of an array of solid-state detectors, including a pair of two-dimensional position-sensitive detectors which determine individual particle trajectories, thereby leading to significant improvement in isotope resolution over previous cosmic-ray instruments. This study includes particles stopping in the last four analyzed detectors of HIST, corresponding to an energy range of $83-284 \mathrm{MeV}$ per nucleon for ${ }^{56} \mathrm{Fe}$ nuclei.

The method of resolving isotopes in HIST has been discussed by Mewald et al. $(1979 b, 1980)$. In this study, we used the outputs of the last three triggered detectors to make two determinations of the charge $z$ and mass $M$. Those events for which the two mass determinations were consistent to within $5 \%$ were accepted for further analysis, with the outputs of the last two detectors used for a best estimate of $M$. Only one event was eliminated by this consistency test.

Figure $1 a$ shows an $\mathrm{Fe}$ mass histogram obtained in accelerator calibrations of the flight HIST instrument with an ${ }^{56} \mathrm{Fe}$ beam at the Lawrence Berkeley Laboratory Bevalac. The Bevalac beam contained, in addition to ${ }^{56} \mathrm{Fe}, \sim 3 \%{ }^{54} \mathrm{Fe}$ and other $\mathrm{Fe}$ isotopes produced in material used to reduce the beam energy.

Figure $1 b$ shows cosmic-ray data from the same 


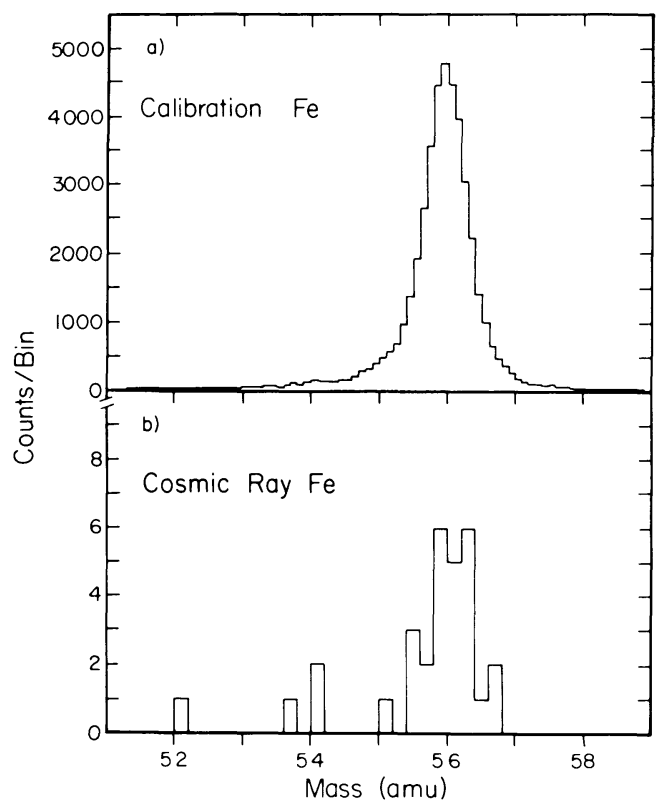

FIG. 1.-Mass histograms of (a) Bevalac calibration data (42,915 events) and (b) cosmic-ray data (30 events), for 83-284 $\mathrm{MeV}$ per nucleon $\mathrm{Fe}$ isotopes. For the single event at ${ }^{52} \mathrm{Fe}$, we have three separate and consistent mass determinations. Since this event most likely resulted from a nuclear reaction earlier in the detector stack, it was not included in the analysis.

energy range. Both data sets in Figure 1 were analyzed in exactly the same manner, using identical selection criteria and instrument calibrations. Comparisons of Bevalac and cosmic-ray data for ${ }^{20} \mathrm{Ne},{ }^{24} \mathrm{Mg}$, and ${ }^{28} \mathrm{Si}$ (Mewaldt et al. 1980) verify that the instrument calibration was the same before and after launch. The Bevalac data, therefore, fix absolutely the mass scale. Both the calibration and the cosmic-ray distributions are dominated by a well-resolved peak at ${ }^{56} \mathrm{Fe}$. There is also evidence for ${ }^{54} \mathrm{Fe}$ in the flight data, but no evidence for iron isotopes heavier than ${ }^{56} \mathrm{Fe}$. The mass resolution in the calibration data is $\sigma_{m}=0.32 \mathrm{amu}$, while in the flight data, $\sigma_{m}=0.37 \pm 0.05 \mathrm{amu}$ for the ${ }^{56} \mathrm{Fe}$ peak. To our knowledge, this is the first cosmic-ray $\mathrm{Fe}$ isotope measurement with a directly calibrated absolute mass scale.

Using the method of maximum likelihood, we have determined best estimates of the fractional isotopic abundances and the $68 \%$ confidence intervals consistent with our observations. Since we estimate that less than $1 \%$ of incident ${ }^{56} \mathrm{Fe}$ nuclei interact in our telescope to produce ${ }^{54} \mathrm{Fe}$, we have applied no correction for this effect. Table 1 summarizes the observed isotopic composition at $1 \mathrm{AU}$. In Figure 2 we compare our observations with recent balloon measurements, and with the expected isotopic distribution resulting from a solar system source.

Within the quoted uncertainties, the measurements in Figure 2 are consistent with each other, and with the expected composition resulting from a cosmic-ray source of solar-system isotopic composition. They show no evidence for the large contributions of ${ }^{58} \mathrm{Fe}$ reported
TABLE 1

Fe Isotope Composition

\begin{tabular}{cccc}
\hline \hline Isotope & Observed $(\%)^{\mathrm{b}}$ & $\begin{array}{c}\text { Cosmic Ray } \\
\text { Source }(\%)^{\mathrm{b}}\end{array}$ & $\begin{array}{c}\text { Solar } \\
\text { System }(\%)^{\mathrm{c}}\end{array}$ \\
\hline${ }^{54} \mathrm{Fe} \ldots \ldots \ldots$ & $10(+8,-4)$ & $9(+8,-5)$ & 5.8 \\
${ }^{55} \mathrm{Fe} \ldots \ldots$ & $\leq 10$ & $\leq 7^{\mathrm{d}}$ & 0 \\
${ }^{56} \mathrm{Fe} \ldots \ldots$ & $90(+0,-15)$ & $91(+5,-11)$ & 91.7 \\
${ }^{57} \mathrm{Fe} \ldots \ldots$ & $\leq 8$ & $\leq 8$ & 2.2 \\
${ }^{58} \mathrm{Fe} \ldots \ldots$ & $\leq 6$ & $\leq 6$ & 0.3
\end{tabular}

a Percentage of total iron.

b $68 \%$ confidence intervals or $84 \%$ confidence limits.

c Cameron 1973.

${ }^{\mathrm{d}}$ Assuming ${ }^{55} \mathrm{Fe}$ does not decay.

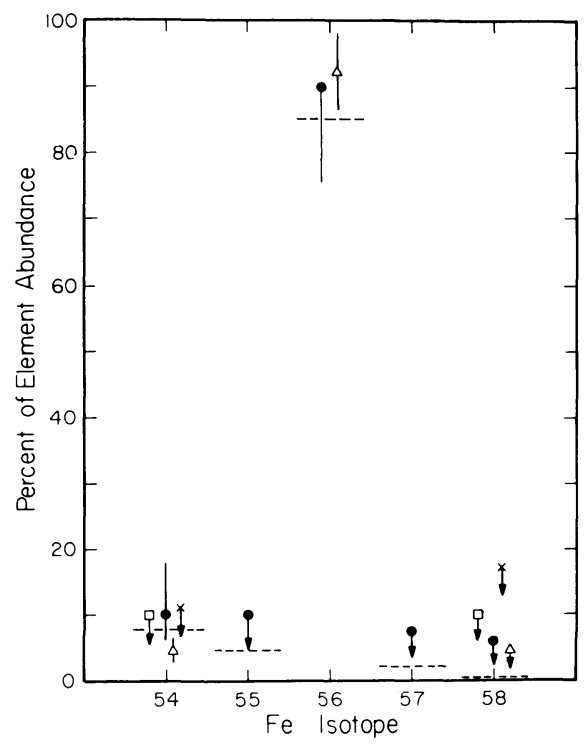

FIG. 2.-A comparison of $\mathrm{Fe}$ isotopic abundances by percent. References for the measurements: solid circles, this work; squares, Tarlé, Ahlen, and Cartwright 1979; triangles, Webber, Kish, and Simpson 1979; crosses, Young 1979. The Webber, Kish, and Simpson (1979) isotope fractions do not take into account possible finite fluxes of ${ }^{55} \mathrm{Fe}$ and ${ }^{57} \mathrm{Fe}$, and so their ${ }^{56} \mathrm{Fe}$ abundance should probably be regarded as ${ }^{55} \mathrm{Fe}+{ }^{56} \mathrm{Fe}+{ }^{57} \mathrm{Fe}$. The dotted lines show the calculated abundances at $1 \mathrm{AU}$ for a solar-system source composition, assuming that all ${ }^{55} \mathrm{Fe}$ produced during propagation survives.

by some earlier experiments with apparently poorer resolution, most recently by Simpson et al. (1977), or for large contributions of ${ }^{54} \mathrm{Fe}(>25 \%)$ that have been reported (see, e.g., the summary of earlier work by Enge 1977). It should be noted that the expected percentage of $\mathrm{Fe}$ nuclei suffering a nuclear interaction within our instrument $(\sim 11 \%)$ is more than 5 times smaller than in the balloon experiments. In addition, no atmospheric corrections are necessary.

\section{COSMIC-RAY SOURCE COMPOSITION}

We have derived the cosmic-ray source composition consistent with our observations by performing interstellar propagation and solar modulation calculations. 
As in Mewaldt et al. (1980), we assumed a standard leaky-box propagation model with an escape mean free path of $\lambda=5.5 \mathrm{~g} \mathrm{~cm}^{-2}$ and used the semi-empirical cross sections of Silberberg, Tsao, and Shapiro (1976). Solar modulation effects on the isotopic composition $(\leq 6 \%)$ were computed for a level of modulation characterized by the energy-loss parameter $\Phi=300 \mathrm{MeV}$ per nucleon. Table 1 includes the source composition of iron isotopes that we derive with their $68 \%$ confidence intervals. Note that propagation effects on the ${ }^{56} \mathrm{Fe},{ }^{57} \mathrm{Fe}$, and ${ }^{58} \mathrm{Fe}$ abundances are essentially negligible (see, e.g., Stone and Wiedenbeck 1979), since there are no abundant nuclei heavier than ${ }^{56} \mathrm{Fe}$. On the other hand, some ${ }^{54} \mathrm{Fe}$ and ${ }^{55} \mathrm{Fe}$ are produced during propagation by the breakup of ${ }^{56} \mathrm{Fe}$. The source composition that we derive is relatively insensitive to the assumed values of $\lambda$ and $\Phi$. For $\lambda=4.5-6.5 \mathrm{~g} \mathrm{~cm}^{-2}$ and $\Phi=200-400 \mathrm{MeV}$ per nucleon, the ${ }^{55} \mathrm{Fe}$, ${ }^{57} \mathrm{Fe}$, and ${ }^{58} \mathrm{Fe}$ limits in Table 1 are unaffected, while the ${ }^{54} \mathrm{Fe}$ and ${ }^{56} \mathrm{Fe}$ limits change by at most $1 \%$.

Also shown in Table 1 is the solar-system isotopic composition of $\mathrm{Fe}$ (Cameron 1973). We see no evidence for a cosmic-ray source composition unlike that of the solar system, a conclusion also reached by Tarlé, Ahlen, and Cartwright (1979); Webber, Kish, and Simpson (1979); and Young (1979). It is evident, however, that high-resolution measurements with significantly better statistics will be needed for accurate determination of the ${ }^{54} \mathrm{Fe},{ }^{57} \mathrm{Fe}$, and ${ }^{58} \mathrm{Fe}$ source abundances.

\section{DISCUSSION}

The nucleosynthesis of $\mathrm{Fe}$ is thought to take place in high-temperature, high-density regions of massive stars under conditions of nuclear-statistical equilibrium (e-process). The isotope distribution of the Fe-peak elements ( $\mathrm{Cr}$ through $\mathrm{Ni}$ ) that results is a sensitive function of the neutron excess $\eta=\left(n_{n}-n_{p}\right) /\left(n_{n}+\right.$ $\left.n_{p}\right)$, where $n_{n}$ and $n_{p}$ are the neutron and proton densities of the stellar zone where nucleosynthesis occurs. Hainebach et al. (1974) concluded that the solar-system iron-peak elements are made up of at least two components characterized by different values of $\eta$. In their two-zone solution, a low- $\eta$ component $(\eta \approx 0.004)$ contributes $\sim 97 \%$ of solar-system $\mathrm{Fe}$ and includes ${ }^{54} \mathrm{Fe}$, ${ }^{56} \mathrm{Fe}$, and ${ }^{57} \mathrm{Fe}$ with relative abundances in agreement with the observed solar-system composition. Under these conditions, ${ }^{56} \mathrm{Fe}$ and ${ }^{57} \mathrm{Fe}$ were actually synthesized as ${ }^{56} \mathrm{Ni}$ and ${ }^{57} \mathrm{Ni}$. A smaller contribution $(\sim 3 \%)$ from a high- $\eta$ zone $(\eta=0.077)$ is required to reproduce the observed ${ }^{58} \mathrm{Fe}$ abundance. It would be surprising if cosmic-ray Fe consisted of exactly the same mixture of the same two components, since cosmic rays and solar-system material might well originate in stars of different masses (Hainebach, Norman, and Schramm 1976), and/or at different stages of the evolution of the galaxy.

Woosley (1976) and Hainebach, Norman, and Schramm (1976) have considered the implications of nonsolar isotopic ratios for cosmic-ray Fe. Figure 3, adapted from Woosley (1976), shows calculated isotope mass-fraction ratios resulting from $e$-process calcula-

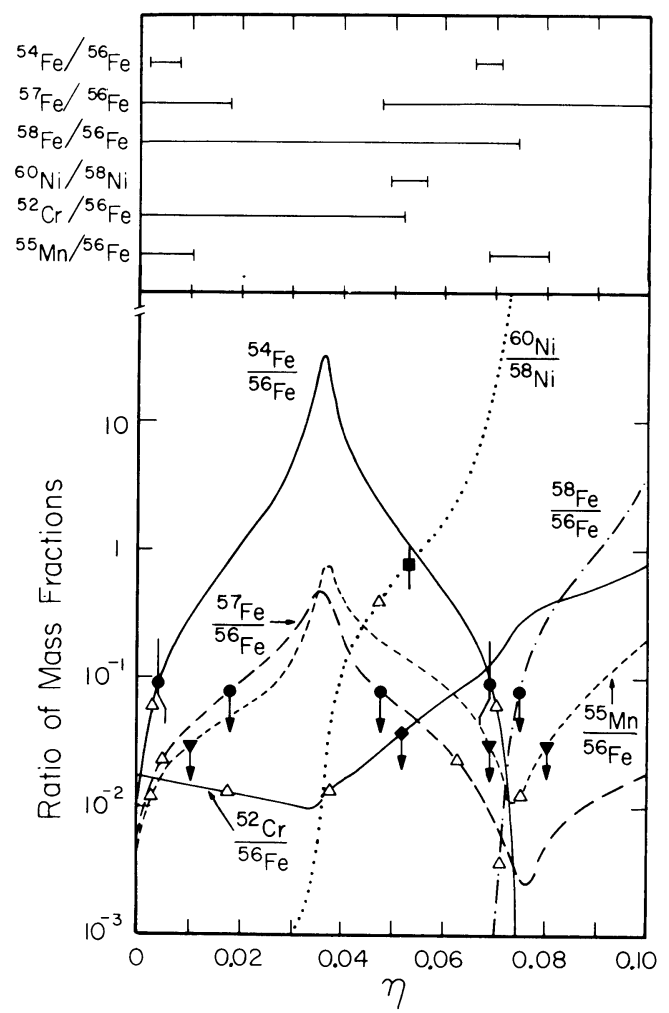

FIG. 3.-Isotope mass fraction ratios as a function of the neutron excess $\eta$. The curves are from calculations by Hainebach et al. (1974). The open triangles are the solar-system abundances after Cameron (1973). The cosmic-ray Fe measurements (solid circles) are from this work only, while the ${ }^{60} \mathrm{Ni} /{ }^{58} \mathrm{Ni}$ point is based on Tarlé, Ahlen, and Cartwright (1979) and on Young (1979) We show also the conservative limits ${ }^{52} \mathrm{Cr} /{ }^{56} \mathrm{Fe}<0.04$ and ${ }^{55} \mathrm{Mn} /$ ${ }^{56} \mathrm{Fe} \leq 0.03$, based on the cosmic-ray source elemental ratios $\mathrm{Cr} / \mathrm{Fe} \leq 0.03$ and $\mathrm{Mn} / \mathrm{Fe} \leq 0.02$ (Garcia-Munoz, Mason, and Simpson 1977). In the top panel, regions of $\eta$ consistent with the cosmic-ray observations are indicated.

tions (Hainebach et al. 1974) as a function of the neutron excess $\eta$. The ${ }^{55} \mathrm{Mn} /{ }^{56} \mathrm{Fe}$ curves assume that all ${ }^{55} \mathrm{Fe}$ produced in the source decays to ${ }^{55} \mathrm{Mn}$ by electron capture (half-life $=2.6 \mathrm{yr}$.). Also shown are measurements of these ratios for the solar system (Cameron 1973) and for cosmic rays. Note that the cosmic-ray ratios for the $\mathrm{Fe}$ isotopes and for ${ }^{55} \mathrm{Mn} /{ }^{56} \mathrm{Fe}$ are consistent with either a "high- $\eta$ " $(\sim 0.07)$ or a "low- $\eta$ " (0.002-0.007) solution, neither of which reproduces the ${ }^{60} \mathrm{Ni} /{ }^{58} \mathrm{Ni}$ observations. The ${ }^{52} \mathrm{Cr} /{ }^{56} \mathrm{Fe}$ observations require $\eta \leq 0.05$ (see also Young 1979). Tarlé, Ahlen, and Cartwright (1979) concluded from their Fe and $\mathrm{Ni}$ isotope observations that the cosmic rays required contributions from more than one $e$-process zone for production, much as Hainebach et al. (1974) concluded for the solar system. Alternatively, the cosmic-ray source may represent a mixture of a single $e$-process zone and some other nucleosynthetic process which contributes, for example, to the ${ }^{60} \mathrm{Ni}$ abundance.

In either case, it is of interest to determine the maximum contribution a given $e$-process zone can make to the cosmic-ray source material. From a comparison 
of the measured and calculated isotopic ratios, such as those in Figure 3, we have computed the maximum allowed percentage of cosmic-ray ${ }^{56} \mathrm{Fe}$ that can derive from a zone with a particular value of $\eta$, as shown in Figure 4 . In calculating the curves in Figure 4 , we reasoned that if a given zone produces more of a specific isotope relative to ${ }^{56} \mathrm{Fe}$ than is observed, then that zone can make only a limited contribution to the total production of ${ }^{56} \mathrm{Fe}$. For example, note in Figure 3 that the $e$-process calculations give ${ }^{58} \mathrm{Fe} /{ }^{56} \mathrm{Fe} \approx 1$ at $\eta=$ 0.09 , while the cosmic-ray upper limit is ${ }^{58} \mathrm{Fe} /{ }^{56} \mathrm{Fe} \leq$ 0.08 . It follows that no more than $8 \%$ of the ${ }^{56} \mathrm{Fe}$ can derive from this $e$-process zone, as is indicated by the ${ }^{58} \mathrm{Fe}$ line at $\eta=0.09$ in Figure 4 . A larger contribution would result in too much ${ }^{58} \mathrm{Fe}$, independent of the isotopic composition of other material with which the $\eta=0.09$ zone is subsequently mixed. In general, for a specific isotopic ratio $(i)$ with observed upper limit $R_{i}$ in the cosmic rays, the maximum allowed contribution for a given $\eta$ is given by $F_{i}(\eta)=100 R_{i} / S_{i}(\eta)$, where $S_{i}(\eta)$ is the calculated $e$-process ratio such as shown in Figure 3. For the $\mathrm{Ni}$ isotope comparison we have used the conservative limits ${ }^{58} \mathrm{Ni} /{ }^{56} \mathrm{Fe} \leq 0.05$ and ${ }^{60} \mathrm{Ni} /$ ${ }^{56} \mathrm{Fe} \leq 0.05$ based on the cosmic-ray ratio $\mathrm{Ni} / \mathrm{Fe}=$ $0.042 \pm 0.007$ (Lezniak and Webber 1978).

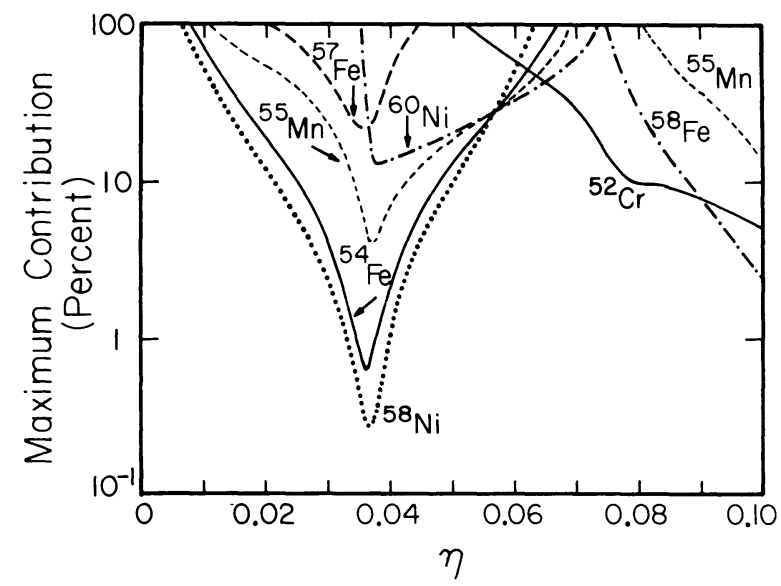

FIG. 4.-The maximum allowed percentage of the ${ }^{66} \mathrm{Fe}$ source abundance that can derive from an $e$-process with any particular value of $\eta$, as constrained by the cosmic-ray source abundances of the indicated isotopes relative to ${ }^{56} \mathrm{Fe}$. The lower bound of the envelope of all the individual curves limits the ${ }^{56} \mathrm{Fe}$ contribution from any given $\eta$.
In Figure 4 it is the lower bound of all the individual curves that limits the ${ }^{56} \mathrm{Fe}$ contribution from any given $\eta$. For example, for $0.07 \leq \eta \leq 0.09$ the ${ }^{52} \mathrm{Cr} /{ }^{56} \mathrm{Fe}$ ratio limits the contribution, while for $0.01 \leq \eta \leq 0.05$, ${ }^{58} \mathrm{Ni} /{ }^{56} \mathrm{Fe}$ imposes the most restrictive $\widetilde{l i m i t s}$. The following conclusions can be drawn from Figure 4. If a single $e$-process zone produces $100 \%$ of cosmic ray ${ }^{56} \mathrm{Fe}$, that zone must have $\eta \leq 0.006$. In this case the cosmic ray ${ }^{60} \mathrm{Ni}$ must be produced by some mechanism other than the $e$-process (see Fig. 3). If only two $e$-process zones contribute to ${ }^{56} \mathrm{Fe}$ synthesis, the dominant zone must have $\eta \leq 0.01$, since for $0.01 \leq \eta \leq 0.1$ the maximum allowed contribution is $\leq 40 \%$. If three or more zones are involved, the contribution from a given $\eta$ is still limited by the bounds in Figure 4 , but it is conceivable that none of the individual contributions is $\geq 50 \%$. In general, only for zones with $\eta \lesssim 0.01$ can the contribution to ${ }^{56} \mathrm{Fe}$ be $\geq 50 \%$ and still satisfy all isotopic constraints. Hainebach et al. (1974) have reached similar conclusions in treating the synthesis of the solar-system iron-peak nuclei. Note, however, that the range $\eta \leq 0.01$ still allows for significant differences in the cosmic-ray and solar-system isotope distribution.

For example, the evidence for enhanced abundances of the neutron-rich isotopes of $\mathrm{Ne}$ (see, e.g., GarciaMunoz, Simpson, and Wefel 1979; Greiner et al. 1979; Mewaldt et al. 1979a, 1980) and possibly Mg (Mewaldt et al. 1979a, 1980) in cosmic rays may be interpreted as due to $\eta$ (or metallicity) values somewhat greater than those that characterize solar-system material, possibly due to galactic evolution effects (Woosley and Weaver 1979). This might suggest an $e$-process $\eta$ value of $\leq 0.004$, not incompatible with the present cosmicray ${ }^{\sim} \mathrm{Fe}$ isotope observations. Further observations should establish whether the neutron-rich character of cosmic-ray $\mathrm{Ne}$ extends to heavier elements, including $\mathrm{Fe}$, thereby providing possible evidence for ongoing nucleosynthesis in the galaxy.

W. E. Althouse, A. C. Cummings, and T. L. Garrard made significant contributions to the design and development of HIST. We are grateful to the Heckman/ Greiner group, including Hank Crawford, and the Bevalac staff at Lawrence Berkeley Laboratory for making the heavy-ion calibrations possible. This work was supported in part by NASA under contract NAS520721 and grant NGR 05-002-160.

\section{REFERENCES}

Althouse, W. E., Cummings, A. C., Garrard, T. L., Mewaldt, R. A., Stone, E. C., and Vogt, R. E. 1978, Geoscience Electronics, 16, 204.

Cameron, A. G. W. 1973, Space Sci. Rev., 15, 121.

Dietrich, W. F., and Simpson, J. A. 1979, Ap. J. (Letters), 231, L91.

Enge, W. 1977, Nucl. Instr. Methods, 147, 211.

Garcia-Munoz, M., Mason, G. M., and Simpson, J. A. 1977, 15th

Internat. Cosmic Ray Conf. (Plovdiv), 1, 224.

Garcia-Munoz, M., Simpson, J. A., and Wefel, J. P. 1979, A p. J. (Letters), 232, L95.
Greiner, D. E., Wiedenbeck, M. E., Bieser, F. S., Crawford, H. J., Heckman, H. H., and Lindstrom, P. J. 1979, 16th Internat. Cosmic Ray Conf. (Kyoto), 1, 418.

Hainebach, K. L., Clayton, D. D., Arnett, W. D., and Woosley, S. E. 1974, Ap. J., 193, 157.

Hainebach, K. L., Norman, E. B., and Schramm, D. N. 1976, Ap. J., 203, 245.

Lezniak, J. A., and Webber, W. R. 1978, Ap. J., 233, 676.

- 1980, A p. J. (Letters), in press.

Mewaldt, R. A., Spalding, J. D., Stone, E. C., and Vogt, R. E. 1979a, 16th Internat. Cosmic Ray Conf. (Kyoto), in press. 
1979b, Ap.J. (Lellers), 231, L97.

$1980, A$ p. J. (Letlers), in press.

Meyer, J. P. 1975, 14th Internat. Cosmic Ray Conf. (Munich: Max Planck-Institut für Extraterrestrische Physik), 11, 3698.

Silberberg, R., Tsao, C. H., and Shapiro, M. M. 1976, in Spallation Nuclear Reactions and Their A pplications, ed. B. S. P. Shen and M. Merker (Dordrecht: Reidel), p. 49.

Simpson, G. A., Kish, J. C., Lezniak, J. A., and Webber, W. R. 1977, Ap. L.etters, 19, 3.

Stone, E. C., and Wiedenbeck, M. E. 1979, Ap. J., 231, 606.

Tarlé, G., Ahlen, S. P., and Cartwright, B. G. 1979, Ap. J., 230, 607 .
Tarlé, G., Ahlen, S. P., Cartwright, B. G., and Solarz, M. 1979, 16th Internat. Cosmic Ray Conf. (Kyoto), 1, 455.

Waddington, C. J. 1977, 15th Internat. Cosmic Ray Conf. (Plovdiv) $10,168$.

Webber, W. R., Kish, J. C., and Simpson, G. A. 1979, 16th Internat. Cosmic Ray Conf. (Kyoto), 1, 430.

Woosley, S. E. 1976, Ap. Space Sci., 39, 103

Woosley, S. E., and Weaver, T. A. 1979, UCRL preprint, No. 83321.

Young, J. S. 1979, Ph.D. thesis, University of Minnesota, Minneapolis.

R. A. Mewaldt, J. D. Spalding, E. C. Stone, and R. E. Vogt: Downs Laboratory of Physics, California Institute of Technology, Pasadena, CA 91125 